\title{
Uma Abordagem Gamificada para o Ensino de Programação Orientada a Objetos
}

\author{
Karen da Silva Figueiredo, Jivago Medeiros Ribeiro, Raphael Souza, Vinicius \\ Raniero Angelo
}

\author{
Instituto de Computação - Universidade Federal do Mato Grosso (UFMT) \\ Cuiabá, Mato Grosso, Brasil \\ \{karen, jivago, raphael\}@ic.ufmt.br, viniciusufegmail.com
}

\begin{abstract}
The application of game design elements in unrelated game contexts is a current practice that is being used in computing in both industry and education scenarios. The use of gamification to teach programming could be seen as a relevant and powerful contemporary strategy to attract students' attention. This paper proposes a gamified approach to teach object-oriented programming in order to engage and motivate students. It also presents the design of a game and the experience of its application in Algorithms III discipline.
\end{abstract}

Resumo. A aplicação de elementos de design de jogos em contextos não relacionados a jogos é uma prática atual que está sendo explorada na área de computação, nos cenários da indústria e da educação. A gamificação no ensino de conteúdos e disciplinas de programação pode ser vista como uma estratégia contemporânea relevante e poderosa para atrair a atenção dos alunos. Este trabalho propõe uma abordagem gamificada para o ensino de programação orientada a objetos com o objetivo de engajar e motivar os alunos, apresentando o design de um jogo e a experiência da sua aplicação na disciplina Algoritmos III.

\section{Introdução}

A gamificação (do inglês "gamification") é uma técnica emergente na indústria que pode ser definida como o "uso de elementos de design de jogos em contextos não relacionados a jogos" [Deterding et al. 2011]. Alguns dos contextos cotidianos nos quais a gamificação pode ser observada são os programas de milhagens de companhias aéreas, aplicativos de competição para redes sociais e programas de fidelidade de restaurantes.

$\mathrm{Na}$ educação e no cenário acadêmico, a gamificação ainda é novidade e os principais trabalhos sobre o tema estão em sua maioria concentrados na década atual [Borges et al. 2013]. O uso da gamificação vem crescendo especialmente na educação formal e nos programas de treinamentos coorporativos com o intuito de engajar alunos em uma experiência de aprendizagem mais imersiva [Lee and Hammer 2011].

No ensino de computação, o uso da gamificação como ferramenta motivacional e instrucional tornou-se assunto popular. Pesquisas mostram que a gamificação já foi utilizada: no ensino de programação introdutória e em conteúdos mais avançados como 
Inteligência Artificial e Engenharia de Software; como elo entre computação e artes em programas interdisciplinares; e como ferramenta motivadora para atrair alunos para cursos de computação e diminuir a evasão [Kumar and Khurana 2012]. No contexto nacional da área, muitas pesquisas estão concentradas na utilização de jogos sérios e os primeiros passos estão sendo dados no caminho da gamificação, e.g. [Bitencourt 2014, Jucá et al. 2014, Andrade and Canese 2013].

No últimos anos, a Programação Orientada a Objetos (POO) mostrou-se o paradigma de programação mais influente. Quase todos os cursos da área de computação incluem a POO como parte do seu currículo [Beck and Cunningham 1989]. Entretanto, ensinar este importante paradigma ainda é difícil, principalmente quando ele é incluído após a programação procedural e o aluno precisar "abandonar" o controle que ele conhece com o paradigma procedural e confiar no conhecimento da POO [COOPER et al. 2003]. Assim, a criação e discussão de técnicas e metodologias de ensino que despertem o interesse do aluno no processo de ensino-aprendizagem da POO são de grande interesse dos educadores da área [Kölling 1999].

O objetivo deste trabalho é descrever o projeto de uma abordagem gamificada para o ensino de POO a fim de engajar e motivar os alunos, apresentando o design proposto para a disciplina Algoritmos III e relatando os resultados dessa experiência. Assim, este trabalho está organizado da seguinte forma: os conceitos necessários relacionados à gamificação para o entendimento do trabalho são discutidos na Seção 2, o design da gamificação proposta e os seus resultados são apresentados na Seção 3 e, por fim, as conclusões e trabalhos futuros são traçados na Seção 4.

\section{Gamificação, Educação e Design}

Segundo o autor do livro "The gamification of learning and instruction" [Kapp 2012], principal obra no tema, a gamificação pode ser utilizada para promover a aprendizagem, reciclando um conteúdo tipicamente apresentado em uma aula tradicional presencial ou a distância por meio da inclusão de elementos de jogos, criando uma oportunidade de aprendizagem gamificada no formato de um jogo. Porém, para que a gamificação seja capaz de fornecer uma camada de interesse a mais no ensino, é importante que os elementos do jogo estejam interconectados, i.e. "cada parte do jogo deve estar conectada a outra parte do jogo" [Kapp 2012]. Os elementos de jogos sozinhos não são suficientes para transformar uma experiência comum ou entediante em uma experiência nova e engajadora, embora sejam peças fundamentais na construção do processo de gamificação.

Kapp (2012) define alguns elementos de jogos que podem ser empregados no design da gamificação para o ensino. Os elementos que são utilizados na proposta deste trabalho são descritos brevemente a seguir:

- Estado ganhador: uma ou mais condições que quando alcançadas definem o ganhador do jogo. $\mathrm{O}$ estado ganhador precisa estar claramente definido para os jogadores. As condições são definidas com base em outros elementos do jogo.

- Jogadores: são as pessoas que participam e interagem com o jogo. No cenário da aprendizagem, os jogadores são geralmente os aprendizes/educandos/alunos, muito embora os professores/educadores possam ter papéis de jogadores 
dependendo das abstrações construídas para o jogo.

- Abstração: jogos geralmente envolvem a abstração da realidade, assim como um software. As estórias e signos utilizados no jogo podem carregar tais abstrações. Deve ficar claro para o designer do jogo que uma abstração não é uma réplica.

- Interação: a interação é fundamental em um jogo. A interação pode ocorrer entre jogadores (competindo ou colaborando), com os elementos do jogo e com o conteúdo do jogo.

- Desafios: jogos desafiam jogadores a alcançarem objetivos gerais e/ou específicos de forma direta e/ou indireta. Um jogo se torna entediante quando não há desafio.

- Feedback: elemento que responde às ações dos jogadores. Pode ser positivo ou negativo e estar acompanhado com uma recompensa. O feedback deve ser instantâneo, direto e claro.

- Recompensas: uma sanção positiva recebida por um jogador, usualmente quando o mesmo vence um desafio ou atinge o estado ganhador. Os tipos mais populares de recompensas internas são pontos e medalhas, porém podem existir recompensas externas aos elementos do jogo.

- Pontuações: um ponto é uma recompensa quantitativa e acumulativa que está relacionada à atividades e comportamentos executados pelos jogadores. $\mathrm{O}$ processo de cálculo de uma pontuação, i.e. conjunto de pontos, deve ser definido em regra(s). Uma pontuação também pode ser decrementada, caso o jogo envolva reforço negativo e punições.

- Rankings ou placares: espaço reservado para a publicação das pontuações dos jogadores. Os placares devem estar visíveis para os jogadores. Quando o placar é organizado de forma a destacar o(s) jogador(res) com maior pontuação ele é chamado de ranking.

- Medalhas: uma recompensa qualitativa, geralmente representada por um signo gráfico abstrato, relacionada à atividades e comportamentos executados pelos jogadores, desafios ou outros estados do jogo. O processo de aquisição de uma medalha pode ser definido em regra ou surpresa de acordo com o design do jogo.

- Regras: conjunto de sentenças que definem o jogo. Elas descrevem como a evolução do jogo funciona, o estado ganhador, o ambiente do jogo, a aquisição de recompensas, o que é justo ou não, etc.

Apenas conhecer os elementos de jogos não é o suficiente para elaborar um bom design de gamificação. A gamificação precisa ser projetada com cuidado e uma solução para esta questão é a utilização de um framework de design como o Gamification Design Framework [Werbach and Hunter 2012]. Um design bem projetado mantém os elementos conectados e fortalece o ambiente do jogo. Neste trabalho, os 6 passos do Gamification Design Framework apontados a seguir são utilizados como orientadores para o processo do design do jogo da disciplina.

1. Definir os objetivos: descrever de forma precisa os objetivos da gamificação e a 
relação com os objetivos do negócio/processo que será gamificado.

2. Traçar os comportamentos desejados: definir quais ações os jogadores devem executar e quais comportamentos específicos os jogadores devem apresentar para que os objetivos do passo 1 sejam alcançados.

3. Descrever os jogadores: traçar um perfil das características dos jogadores envolvidos na gamificação.

4. Criar ciclos de atividades: a gamificação é baseada em ciclos de dois níveis diferentes, micro e macro, que precisam ser planejados e definidos neste passo. No nível micro, são estabelecidas as atividades e feedbacks da gamificação e no macro, o processo da jornada e de evolução dos jogadores.

5. Incluir diversão: passo importante no qual são selecionados os elementos de jogos capazes de promover diversão considerando os objetivos, comportamentos, jogadores e atividades do cenário.

6. Implantar os instrumentos apropriados: encontrar as ferramentas apropriadas para a execução da gamificação, sejam plataformas de software ou outros mecanismos.

\section{Gamificação do Curso de Programação Orientada a Objetos}

Este trabalho propõe uma abordagem gamificada para o ensino de POO a fim de engajar e motivar os alunos, apresentando o design proposto para a disciplina Algoritmos III do curso Bacharelado em Sistemas de Informação da Universidade Federal de Mato Grosso (UFMT) e relatando os resultados dessa experiência. $O$ curso possui um total de 8 semestres em regime de créditos semestral, contendo disciplinas obrigatórias e optativas, presenciais e semipresenciais, em sua matriz curricular.

A disciplina Algoritmos III é uma disciplina obrigatória e presencial com carga horária total de $60 \mathrm{~h}$, ofertada para alunos a partir do $3^{\circ}$ semestre do curso. A disciplina possui como pré-requisitos as disciplinas Algoritmos I e Algoritmos II nas quais os alunos vêem conteúdos relacionados à programação procedural. O primeiro contato no curso com POO e o paradigma orientado a objetos acontece na disciplina Algoritmos III. As subseções seguintes descrevem o design da gamificação e os resultados da experiência executada no primeiro semestre de 2014.

\subsection{Design da Gamificação}

O primeiro passo para a gamificação é a etapa de elaboração do design ou projeto do jogo que, no caso do ensino universitário, pode ser desenvolvido em conjunto com a etapa de planejamento de ensino da disciplina. A gamificação, quando bem projetada, pode ajudar alunos a adquirirem habilidades, conhecimentos e competências em um período curto e concentrado de tempo, com uma alta taxa de retenção e memorização eficaz do conteúdo [Kapp 2012], ideal para auxiliar o cenário de um curso semestral com conteúdos novos e complexos como os de Algoritmos III.

O Gamification Design Framework [Werbach and Hunter 2012] foi utilizado como base metodológica para a produção do design da gamificação da disciplina. A seguir, os 6 passos para a gamificação de Algoritmos III são apresentados de acordo 
com as definições do framework dadas na Seção 3.

1. Objetivos: (i) motivar os alunos para aumentarem suas participações nas aulas e atividades propostas e (ii) engajar os alunos no processo de ensino-aprendizagem dos conceitos de POO a fim de alcançarem melhores resultados nas avaliações da disciplina.

2. Comportamentos desejados: para alcançar os objetivos citados, foram incentivados os seguintes comportamentos no alunos: autoconfiança para exporem suas ideias nas aulas; segurança para fazerem perguntas pertinentes aos conteúdos; proatividade na busca por informações; colaboração com os colegas e espaço de aula e comprometimento com prazos de atividades.

3. Jogadores: os jogadores foram os alunos matriculados na disciplina Algoritmos III no semestre 2014-1, a saber: 28 alunos matriculados (2 mulheres e 26 homens), sendo 92\% com idade entre 20 e 29 anos (mínimo de 20, máximo de 44). Todos os alunos tinham alguma experiência prévia com jogos e apenas 2 alunos disseram conhecer o conceito de gamificação. Não foram definidos papéis variados para os jogadores, porém os alunos foram orientados a escolherem um nome de jogador para que o seu desempenho no jogo fosse anonimizado, evitando possíveis embaraçamentos perante os colegas.

4. Ciclos de atividades: os ciclos de atividades que delimitam a estrutura do jogo da disciplina foram definidos temporalmente e por conteúdo. Optou-se por dividir a disciplina em 15 semanas de $4 \mathrm{~h}$ ( 2 aulas de $2 \mathrm{~h}$ por semana). Em cada semana foram ministrados 2 conteúdos programáticos do plano de ensino da disciplina e para cada conteúdo existia uma tarefa a ser desenvolvida pelos alunos.

5. Diversão: os elementos de jogos selecionados para a diversão na gamificação de Algoritmos III foram os descritos na Seção 3. As 15 semanas de aula foram mapeadas como desafios com pontuações a serem alcançados pelos alunos. Os alunos foram recompensados com pontuações positivas e medalhas quando apresentavam os comportamentos desejados traçados em determinado ciclo/semana (nível micro). O feedback da evolução nos desafios, pontos e medalhas do jogador podiam ser visualizados ao longo da disciplina (nível macro). A gamificação da disciplina Algoritmos III foi executada com uma abordagem de interação de jogo competitiva e incluía um ranking por pontos e medalhas individuais dos jogadores. A natureza competitiva da gamificação encoraja muitos a fazerem o seu melhor para atingirem o objetivo de ganhar. $\mathrm{O}$ estado ganhador foi dado pelo jogador com o maior número de pontos ao final da disciplina (o maior número de medalhas seria utilizado como critério de desempate, caso necessário). A recompensa estabelecida para o jogador que alcançasse o estado ganhador foi um certificado produzido junto com a Coordenação de Curso de Sistemas de Informação que confirmava o desempenho de destaque na disciplina. As regras da gamificação foram apresentadas para os alunos no início da disciplina e disponibilizadas para consulta posterior.

6. Instrumentos: o ambiente de jogo da disciplina era composto pelo espaço físico 
das aulas presenciais (laboratório de informática), AVA da disciplina e Plataforma de Gamificação. Os instrumentos utilizados na gamificação foram a Tabela de Pontos, o recurso de Tarefas no AVA e a Plataforma de Gamificação que são descritos com mais detalhes a seguir.

A fim de cobrir o espaço físico das aulas, uma Tabela de Pontos foi elaborada para a atribuição de pontos para os jogadores. Quando um aluno apresentava um dos comportamentos desejados em aula (e.g. realizar uma pergunta, contribuir com uma informação, ajudar um colega), o mesmo recebia 1 ponto. A tabela foi manipulada pelo aluno monitor e pelo professor da disciplina. A Figura 1 mostra uma representação da Tabela de Pontos. Posteriormente, os pontos eram atualizados na Plataforma de Gamificação onde ficavam disponíveis para os jogadores.

\begin{tabular}{|c|c|c|c|c|c|c|}
\hline \multicolumn{3}{|c|}{ Tabela de Pontos } & \multicolumn{2}{|r|}{ Semana:X } & \multicolumn{2}{|c|}{ Data: $\mathrm{XX} / \mathrm{XX} / \mathbf{X X X X}$} \\
\hline Jogador & $\begin{array}{l}\text { Pergunta } \\
\text { Pertinente }\end{array}$ & $\begin{array}{l}\text { Comentário } \\
\text { Interessante }\end{array}$ & $\begin{array}{c}\text { Resposta ao } \\
\text { Professor }\end{array}$ & $\begin{array}{l}\text { Resolução de Exercício } \\
\text { em Sala no Quadro }\end{array}$ & Dica & $\begin{array}{l}\text { Ajuda a um } \\
\text { colega }\end{array}$ \\
\hline Jogador1 & III & & 1 & & & \\
\hline Jogador2 & & 1 & & 1 & & III \\
\hline Jogador3 & II & & & & W4 & \\
\hline
\end{tabular}

Figura 1. Representação da Tabela de Pontos

A UFMT disponibiliza um AVA oficial para as disciplinas (Moodle na versão 1.9), que geralmente já é utilizado pelos professores para comunicação com os alunos, hospedagem de materiais e envio de atividades das disciplinas. $\mathrm{O}$ recurso de Tarefas do AVA foi utilizado como instrumento para a gamificação. As tarefas enviadas dentro do prazo pelos alunos via AVA eram recompensadas com pontos nos desafios semanais e medalhas. Ambos podiam ser consultados na Plataforma de Gamificação.

As medalhas projetadas para a disciplina foram classificadas em dois tipos: medalhas de comportamento, que eram conquistadas ao executar um determinado comportamento em uma frequência $\boldsymbol{n}$ (contabilizada através da Tabela de Pontos); e medalhas temáticas relacionadas a um conteúdo específico da disciplina, que eram conquistadas por enviar no prazo via AVA uma tarefa de determinado conteúdo. Alguns exemplos de medalhas são ilustrados na Figura 2.

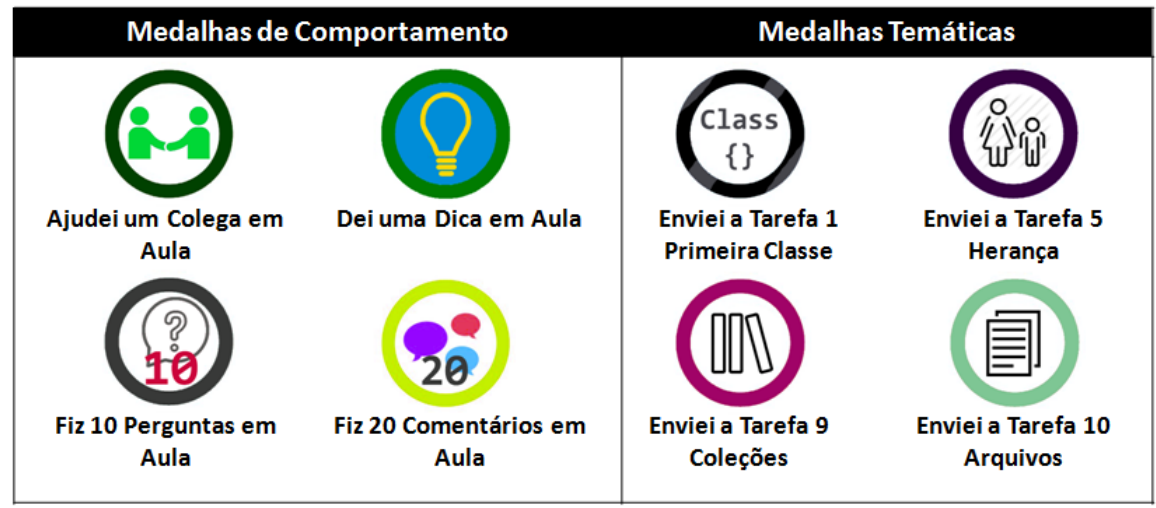

Figura 2. Exemplos de medalhas

A Plataforma de Gamificação foi o principal instrumento utilizado na gamificação de Algoritmos III. Esta plataforma foi desenvolvida especialmente para a 
gamificação da disciplina com o objetivo de apresentar um canal específico de comunicação no qual os alunos pudessem visualizar a evolução do jogo, tendo em vista que a versão do AVA oferecido pela Universidade não apresenta os recursos necessários para tal.

A Plataforma de Gamificação possui duas interfaces: Resultados do Jogo e Perfil do Jogador. A interface Perfil do Jogador (ilustrada na Figura 3) continha as seguintes informações: 1- nome do jogador, 2- pontuação semanal e progresso dos desafios semanais, 3- total de pontos (somatório dos pontos de todas as semanas), 4- medalhas recebidas e total de medalhas.

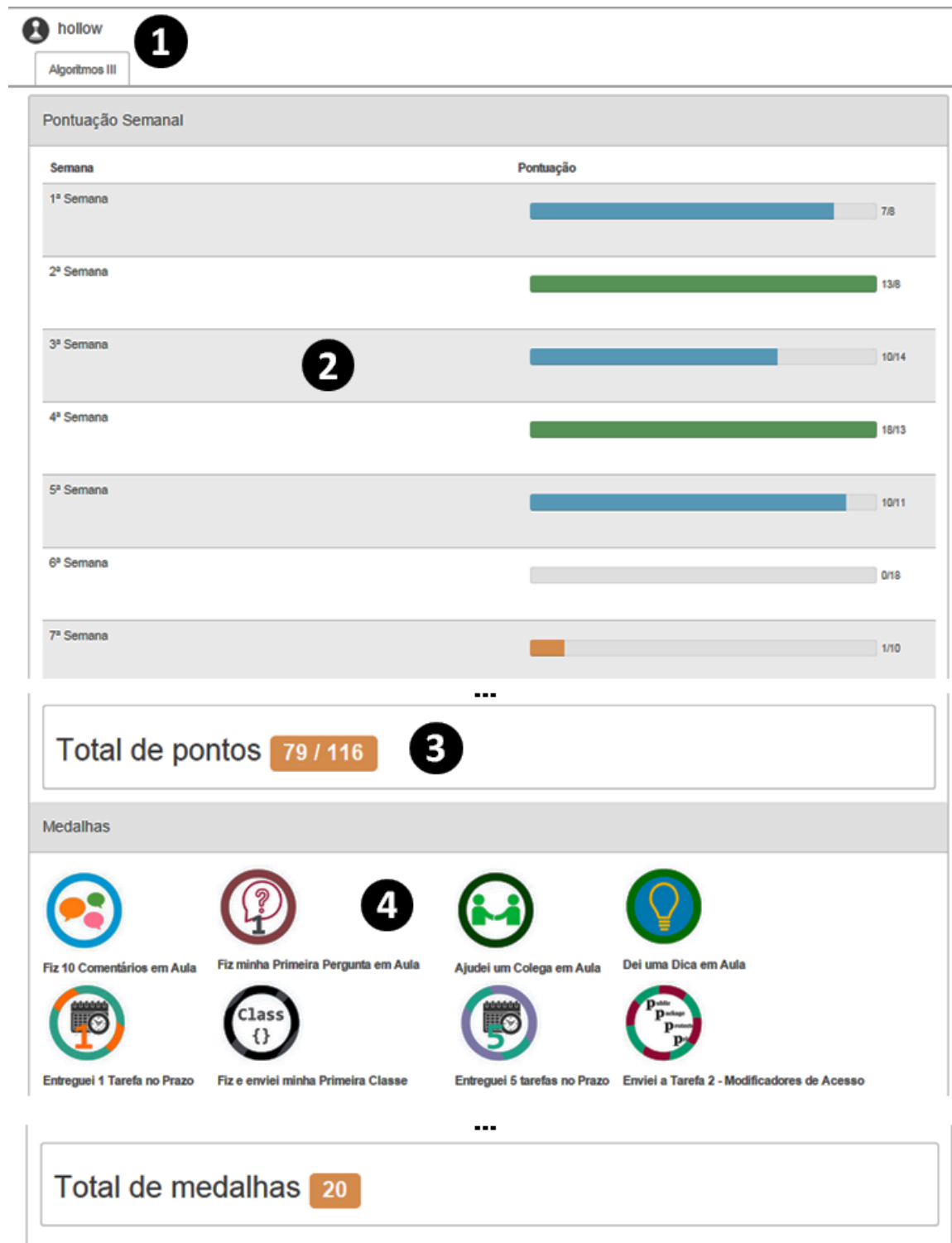

Figura 3. Exemplo resumido da interface Perfil do Jogador

A interface Resultados do Jogo (ilustrada na Figura 4) apresentava os rankings de jogadores com links para os perfis dos mesmos: 1- ranking por pontos e 2-ranking por medalhas. $\mathrm{O}$ jogador no estado ganhador ( $1^{\mathrm{a}}$ posição no ranking por pontos) era destacado em negrito. 
A Plataforma de Gamificação foi constantemente alimentada após cada aula durante o período de execução da gamificação com o intuito de manter o feedback claro e atualizado do estado do jogo para os alunos jogadores.

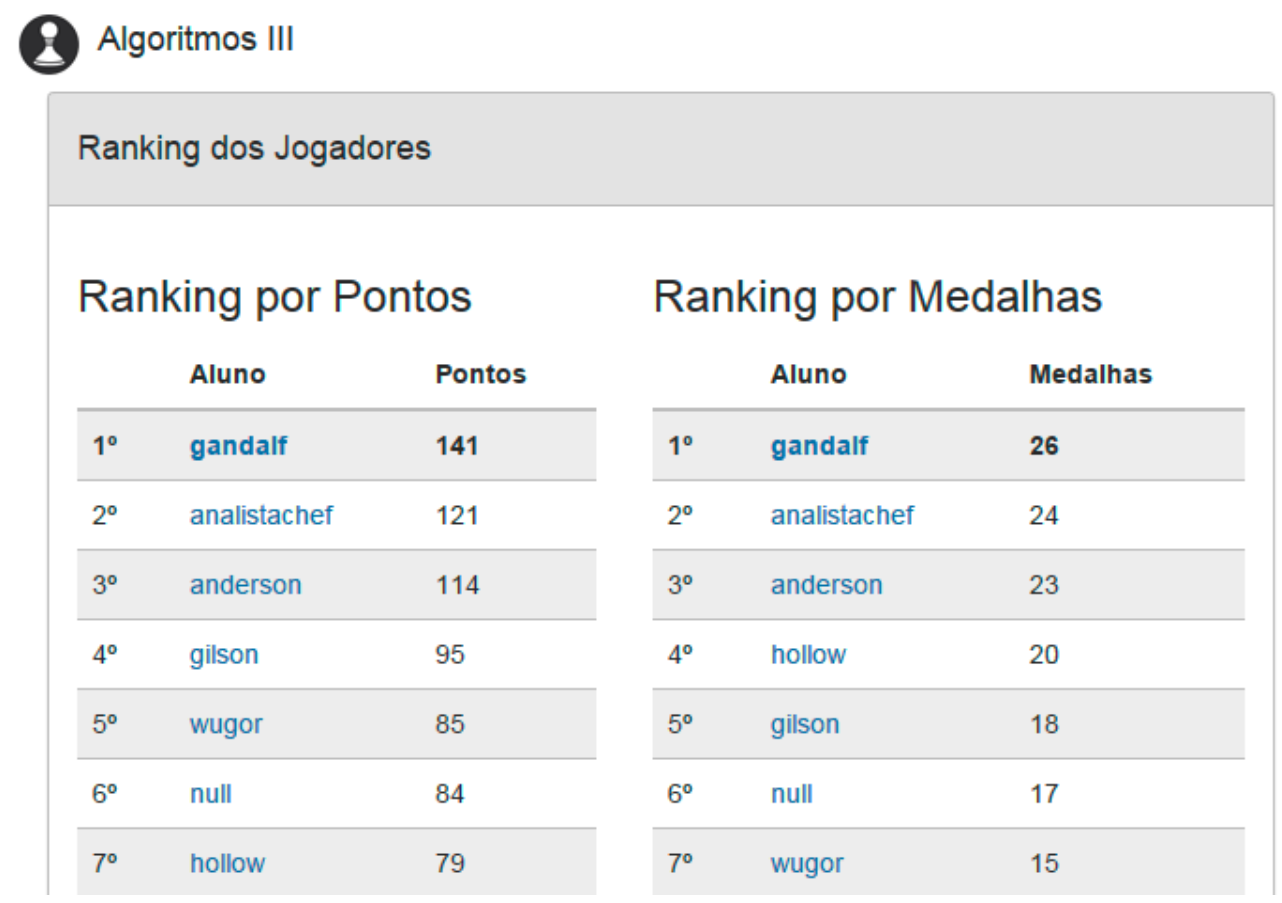

Figura 4. Exemplo resumido da interface Resultados do Jogo

\subsection{Resultados da Experiência}

A fim de verificar os resultados da experiência de gamificação, os dados quantitativos referentes aos resultados das avaliações da disciplina Algoritmos III de 2014-1 do curso de Sistemas de Informação (Turma Gamificada - TG) foram comparados com os dados da disciplina Algoritmos III de 2014-1 do curso de Ciência da Computação (Turma Não Gamificada - TNG) da mesma instituição. Ambas disciplinas possuem a mesma ementa, conteúdos programáticos, carga horária e pré-requisitos. Os planejamentos das disciplinas foram feitos em conjunto por ambos os professores e os mesmos materiais didáticos, AVA e instrumentos de avaliações foram utilizados, diferenciando-se apenas pelo uso da gamificação.

O processo de avaliação das disciplinas foi dado da seguinte forma: 2 provas escritas (P1 e P2), 20 tarefas via AVA $(\mathrm{T})$ e projeto final prático $(\mathrm{PF})$, constituindo a seguinte fórmula para cálculo da nota da disciplina: NOTA $=\left(((\mathrm{P} 1+\mathrm{P} 2) / 2)^{*} 0,4+\right.$ $\left.\left.\mathrm{PF}^{*} 0,4+\left(\sum \mathrm{Tn} / 20\right)^{*} 0,2\right)\right)$. No design da gamificação proposta, nenhum dos elementos de jogos influencia na nota dos alunos da TG. Ter uma baixa pontuação na gamificação não implica diretamente em ter uma baixa nota na disciplina. O conceito da nota não foi amarrado à gamificação, pois jogos devem fornecer um ambiente seguro para que os alunos possam explorar, pensar e experimentar [Kapp 2012].

A TG possuía 28 alunos matriculados, tendo sido descartados os registros de NOTA $=0,0$ de 2 alunos que nunca frequentaram a disciplina. Já a TNG possuía 22 alunos matriculados, descartando 3 registros de NOTA $=0,0$ de alunos que nunca frequentaram a disciplina. A TG apresentou média de 7,4 com desvio padrão de 1,32 e a 
TNG média de 5,48 com desvio padrão de 2,68.

Através da Figura 5, percebe-se que o boxplot da TG apresenta um outlier (registro de nota 4). Para a TNG existem 4 outliers, (registros de notas 0,$45 ; 0,52 ; 0,67 \mathrm{e}$ 1,30). Pode-se observar também que as medianas das duas turmas diferem e que o terceiro quartil da TNG é bem próximo da mediana da TG.

Com o objetivo de comparar as médias das notas das disiciplinas da TG e da TNG foi realizado o teste não paramétrico de Wilcoxon-Mann-Whitney. As seguintes hipóteses foram testadas: H0: As médias de notas das turmas são iguais; H1: As médias de notas das turmas são diferentes. O teste apresentou um p-valor de 0,01307 , que é menor que 0,05 , portanto há indícios para rejeitar a hipótese de que as médias das notas das turmas são iguais ao nível de 5\% de significância, concluindo que a média de notas da TG é maior que a média de notas da TNG.
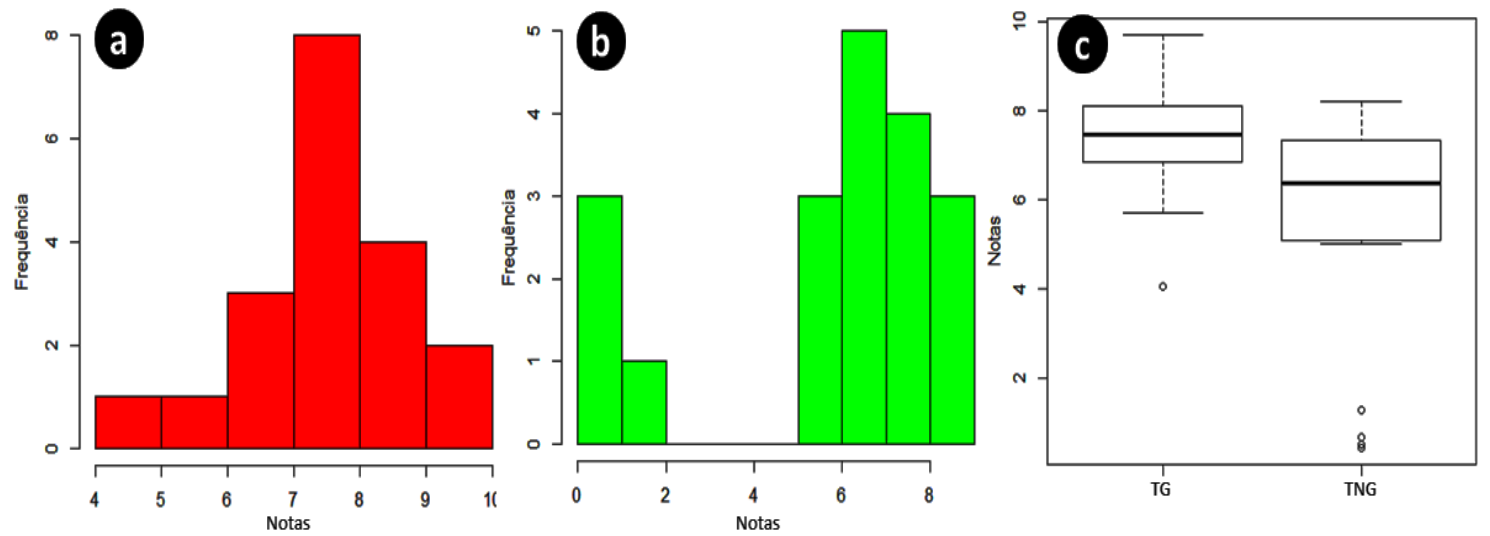

Figura 5. a) Histograma TG, b) Histograma TNG e c) Médias de notas TG e TNG

Considerando os números expostos, é possível afirmar que os objetivos propostos pela gamificação foram alcançados, principalmente no que diz respeito aos melhores resultados nas avaliações da disciplina. A partir da análise isolada dos instrumentos de avaliação, ainda é posível complementar, salientando que a média de desempenho da TG nas tarefas via AVA e no projeto final foram, aproximadamente, $42 \%$ e $25 \%$ respectivamente, maiores que as da $\mathrm{TNG}$, reforçando a participação dos alunos da TG nas atividades propostas da disciplina.

\section{Conclusões}

Este trabalho apresentou uma abordagem gamificada para o ensino de POO através do relato de design de uma gamificação e seus resultados na disciplina Algoritmos III. Apesar da abordagem ter sido aplicada em um cenário específico (disciplina Algoritmos III), o processo de design da gamificação é exposto para ser facilmente adaptado para qualquer disciplina de computação de acordo com a contribuição dos conceitos de gamificação, elementos de jogos e roteiro de design do framework apresentados neste artigo.

Como trabalhos futuros, é possível citar uma análise mais profunda, quantitativamente e qualitativamente, dos dados coletados (considerando o limite de tamanho deste artigo) e a melhoria e ampliação da Plataforma de Gamificação utilizada para permitir a gamificação de outras disciplinas. A aplicação da gamificação no ensino 
de disciplinas de cursos da área de computação pode ser vista como uma das estratégias contemporâneas relevantes e poderosas que são citadas em [Hafeez-Baig et al. 2012] para contribuição na área, tendo em vista que grande parte dos alunos de tais cursos presenciaram uma evolução digital da sociedade que inclui um mercado de jogos cada vez mais presente.

\section{Referências}

Andrade, J. and Canese, M. (2013) "Elementar: Aplicando Gamificação ao Processo de Ensino-Aprendizagem da Lógica Formal”, Conferencias LACLO, v. 4, n. 1.

Beck, K. and Cunningham, W. (1989) "A laboratory for teaching object oriented thinking”, In: ACM Sigplan Notices, ACM, 1989. p. 1-6.

Bitencourt, R. (2014) "Experiência de gamificação do ensino na Licenciatura em Computação no Sertão Pernambucano", In: XIII Simpósio Brasileiro de Games e Entretenimento Digital (SBGames 2014).

Borges, S., et al. (2013) "Gamificação Aplicada à Educação: Um Mapeamento Sistemático", In: XXIV Simpósio Brasileiro de Informática na Educação (SBIE 2013).

Cooper, S. et al. (2003) "Teaching objects-first in introductory computer science", $A C M$ SIGCSE Bulletin, v. 35, n. 1, p. 191-195.

Deterding, S., et al. (2011) "From game design elements to gamefulness: defining gamification", In: Proceedings of the 15th International Academic MindTrek Conference: Envisioning Future Media Environments, ACM, p.9-15.

Hafeez-Baig, A. et al (2012) "Feeding back and enhancing authentic learning in quality course assessment design: locating information systems education in rigorous educational research", In: Proceedings of the 23rd Australasian Conference on Information Systems 2012, ACIS.

Jucá, P. et al. (2014) “Aplicação da Gamificação na Disciplina de Empreendedorismo”, In: XXII Workshop sobre Educação em Computação (WEI 2014).

Kapp, K. (2012) "The gamification of learning and instruction: game-based methods and strategies for training and education”, John Wiley \& Sons.

Kölling, M. (1999) "The problem of teaching object-oriented programming", Journal of Object Oriented Programming, v. 11, n. 8, p. 8-15.

Kumar, B. and Khurana, P. (2012) "Gamification in education-learn computer programming with fun", International Journal of Computers and Distributed Systems, v. 2, n. 1, p. 46-53.

Lee, J. and Hammer, J. (2011) "Gamification in education: What, how, why bother?", Academic Exchange Quarterly, v. 15, n. 2, p. 146.

Werbach, K. and Hunter, D. (2012) "For the win: How game thinking can revolutionize your business", Wharton Digital Press. 